\title{
BILATERAL ERECTOR SPINAE PLANE BLOCKS AS AN ALTERNATIVE TO EPIDURAL IN A PATIENT ON CLOPIDOGREL: A CASE REPORT
}

\author{
C.Prior ${ }^{1}$, A.Knaggs ${ }^{2}$, B.Bellew ${ }^{3}$ \\ ${ }_{1}^{1}$ Anaesthetic registrar, St.Mary's Hospital, Paddington, London \\ ${ }^{2,3}$ Consultant Anaesthetist, St.Mary's Hospital, Paddington, London
}

\section{BACKGROUND}

- Central neuraxial blockade (CNB) techniques have been shown to provide excellent analgesia and reduce mortality and morbidity following high-risk surgery.

- However, they may be contraindicated in patients who are coagulopathic or taking anticoagulant or antiplatelet medications.

- As described in this case, bilateral erector spinae plane block (ESPB) may provide a suitable alternative in certain patients.

\section{CASE REPORT}

- A 63-year-old female patient was diagnosed with persistent ilio-lumbar type II and III endoleaks with a $12 \mathrm{~cm}$ aneurysm sac.

- This occurred following endovascular aneurysm repair for a ruptured infra-renal abdominal aortic aneurysm 3 years previously.

- On pre-operative assessment, she was found to be bedbound with multiple co-morbidities including type 2 diabetes, ischaemic heart disease, previous cadaveric renal transplant for end-stage renal failure, cerebrovascular disease and ankylosing spondylitis.

- She had suffered a non-ST-elevation myocardial infarction 6 months previously and required one year of dual antiplatelet therapy following placement of a drug-eluting stent to the left anterior descending coronary artery.

- A V-POSSUM score was calculated giving a morbidity and mortality risk of $98 \%$ and $58 \%$ respectively. Following an appropriate multi-disciplinary discussion with the patient, a laparotomy via a rooftop incision was planned for exploration and repair of the endoleak.

- Effective analgesia was considered imperative for reducing post-operative morbidity. However, epidural catheter placement was contra-indicated due to dual antiplatelet therapy including clopidogrel. Bilateral erector spinae plane catheter (ESPC) insertion was considered as an alternative analgesic technique.

- Following general anaesthesia, bilateral ESPC were placed with in-plane ultrasound guidance. An initial bolus of $20 \mathrm{mls}$ $0.25 \%$ levobupivacaine was given bilaterally.
- Post-operatively, an infusion of plain $0.125 \%$ levobupivacaine was commenced at $10 \mathrm{mls} /$ hour through each ESPC.

- Following laparotomy and exploration of the aneurysm sac, ligation of lumbar arteries and repair of inferior vena cava was required.

- Multiple blood products including 28 units of packed red cells were administered intra-operatively.

- On day 2 post-operatively, the patient was able to cough comfortably and was successfully extubated.

- The ESPC were removed on day 4 post-operatively.

\section{DISCUSSION}

- As well as providing excellent analgesia, the addition of CNB to general anaesthesia can reduce morbidity following high-risk surgery. Specifically, it has been shown to reduce the incidence of post-operative pneumonia by approximately $30 \%$ !.

- However, due to the risk of epidural haematoma, CNB is contra-indicated in patients requiring some antiplatelet therapies including clopidogrel. Although peripheral nerve block (PNB) is also relatively contraindicated in such patients, the potential benefit of reduced post-operative morbidity in high-risk patients may outweigh the risk of bleeding associated with PNB.

- This risk can be mitigated if PNB is performed by an experienced operator, with the use of ultrasound and colour Doppler to facilitate safe needle trajectory ${ }^{2}$.

- ESPB may be classified as a PNB and has been shown to be efficacious for abdominal surgery ${ }^{3}$.

- As ESPB is a relatively new technique, the risk of haemorrhagic complications is not described in current guidelines ${ }^{4,5}$.

- We suggest that ESPC may provide a safer alternative to epidurals in patients on clopidogrel.

- However, further work is needed to evaluate the risk of haemorrhagic complications in ESPB as well as other PNBs. Subsequent guidelines will help in assessing the risk/benefit balance in high-risk patients.

\section{REFERENCES}

- 'Guay J et al. Neuraxial blockade for the prevention of postoperative mortality and major morbidity: an overview of Cochrane systematic reviews. Cochrane Database of Systematic Reviews 2014, Issue I.Art No.: CDI0108

- $\quad{ }^{2}$ Bellew B. Peripheral nerve blocks in a patient on dual anti-platelet therapy. ESRA Academy 20 I7; 190688

- $\quad{ }^{3}$ Chin $\mathrm{KJ}$ et al. The analgesic efficacy of pre-operative bilateral erector spinae plane (ESP) blocks in patients having ventral hernia repair. Anaesthesia 2017; 72: 452-460

- ${ }^{4}$ Association of Anaesthetists of Great Britain and Ireland, Obstetric Anaesthetists' Association and Regional Anaesthesia uk. Regional anaesthesia and patients with abnormalities of coagulation. Anaesthesia 2013; 68: 966-72

- ${ }^{5}$ American Society of Regional Anaesthesia and Pain Medicine. Interventional Spine and Pain Procedures in Patients on Antiplatelet and Anticoagulant Medications Guidelines. Regional Anaesthesia and Pain Medicine 2015; 40(3):182-212

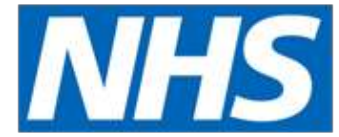

Imperial College Healthcare NHS Trust 